\title{
Simple PV Performance Equations Theoretically Well-Founded on the Single-Diode Model
}

\author{
Efstratios I. Batzelis, Member, IEEE
}

\begin{abstract}
There are several photovoltaic (PV) performance models in the literature, but most of them either employ complex and tedious calculations or require additional measurements apart from datasheet information. In this paper, a new set of performance equations to evaluate the short circuit (SC) current, open circuit (OC) voltage and maximum power point (MPP) at any operating conditions is introduced. The proposed expressions are simple functions of the irradiance and temperature, while they are generally applicable to any crystalline $P V$ module and require only datasheet information as input data. This is achieved by introducing new formulas to determine the irradiance and temperature coefficients that are not provided in the datasheet, thus avoiding empirical constants or additional measurements. The novelty of the performance equations is their solid theoretical background, as they are in excellent agreement with the singlediode PV model, combined with simple and easy application. The proposed PV model is validated and compared to other methods found in the literature through simulations in MATLAB and outdoor measurements on commercial PV modules.
\end{abstract}

Index Terms - Explicit expressions, five parameters, irradiance factors, Lambert $\mathrm{W}$ function, maximum power point (MPP), performance equations, photovoltaic (PV), single-diode model, temperature coefficients.

\section{NOMENCLATURE}

FF Fill factor.

$G \quad$ Incident irradiance (normalized).

$I_{m p}, V_{m p}, P_{m p} \quad$ MPP current, voltage, power.

$I_{p h}, I_{s}, a, R_{s}, R_{s h}$ The five parameters of the single-diode PV model.

$I_{s c}, V_{o c} \quad S C$ current, OC voltage.

SC, OC, MPP Short circuit, open circuit, maximum power point.

STC Standard test conditions $\left(1000 \mathrm{~W} / \mathrm{m}^{2}, T_{0}=298.15 \mathrm{~K}\right)$.

$T, \Delta T, \lambda T \quad$ Cell temperature: absolute value $(\mathrm{K})$, difference $T$ -

$T_{0}(\mathrm{~K})$, ratio $T / T_{0}$ (normalized).

$w \quad$ Auxiliary parameter $W\left\{I_{p h} e / I_{s}\right\}$ (dimensionless).

$W\{x\} \quad$ Lambert $W$ function of $x$.

$X_{0} \quad$ Generic parameter $X$ at STC.

$\alpha_{I s c}, \alpha_{I m p} \quad$ Normalized temperature coefficient of $I_{s c}, I_{m p}\left(\mathrm{~K}^{-1}\right)$.

$\beta_{V o c}, \beta_{V m p} \quad$ Normalized temperature coefficient of $V_{o c}, V_{m p}\left(\mathrm{~K}^{-1}\right)$.

$\delta_{0} \quad$ Irradiance coefficient of $V_{o c}$ (dimensionless).

$\varepsilon_{0}, \varepsilon_{1} \quad$ Irradiance coefficients of $V_{m p}$ (dimensionless).

\section{INTRODUCTION}

$\mathrm{T}^{\mathrm{H}}$ HE term photovoltaic $(P V)$ performance model (or simply $P V$ model) refers to a mathematical model that calculates the electrical response of a PV system at any operating

Manuscript received February 19, 2017; revised April 7, 2017; accepted May 24, 2017.

The author was with the School of Electrical and Computer Engineering, National Technical University of Athens, Athens 15780, Greece. He is now with the Department of Electrical and Electronic Engineering, Imperial College London, London SW7 2AZ, UK (e-mail: stratis.batzelis@ gmail.com). conditions, i.e. incident irradiance $G$ and cell temperature $T$. There are mainly two different types of PV performance models in the literature employing either (a) a detailed circuit model [1]-[10] or (b) simple semi-empirical expressions [2]-[6], [11]-[24]. The former approach adopts an electrical equivalent circuit and permits derivation of the entire $I-V$ characteristic. It provides complete information on the system's operation, but generally requires several implementation steps such as: extraction of the model parameters, translation to actual operating conditions, derivation of the characteristic curve etc. On the other hand, the methods that adopt semi-empirical expressions are straightforward and more easy-to-use, evaluating simple explicit equations in a single step. However, they provide specific only information, such as the short circuit (SC) current, the open circuit (OC) voltage and the maximum power point (MPP), rather than the entire characteristic. The latter methods are the focus of this paper.

There are several PV performance models of this type in the literature [2]-[6], [11]-[16], [18]-[21], [23], [24], as well as in two standards: ASTM E 1036M - 96 [17] and IEC 60891 [22]. A major distinction between these models is whether the information provided in the PV module datasheet suffices for their application [2]-[6], [11], [12] or additional measurements on the study-case PV system are required [13]-[24]. The limitation of the latter methods render them generally much more difficult-to-apply compared to the former ones, especially in simulation studies when there is no PV system available for measurements. Unfortunately, the models derived by wellknown laboratories, such as NREL [13]-[16] and SANDIA [18]-[21], as well as the two abovementioned standards [17], [22] fall into this category. This is because they require irradiance and temperature coefficients, as well as other data usually not available in the datasheet [22], [25]. Therefore, it remains nowadays a popular research topic to derive a mathematical PV model that is at the same time simple, accurate and generally applicable.

Among the abovementioned semi-empirical models that rely solely on datasheet information, only the models in [2], [4]-[6], [11], [12] provide all essential information required for a PV energy yield study: the SC current, the OC voltage and the MPP voltage and current, denoted hereafter as the three key operating points. If these are known for a particular PV system, then all other energy yield parameters are readily derived, such as the maximum power or fill factor $(\mathrm{FF})$.

However, the simplicity of the aforementioned models comes at the cost of reduced accuracy, as they either rely on empirical observations or consider questionable assumptions. Specifically, the equations proposed in [11] are based on 
empirical fill-factor-relations that employ empirical constants and lack theoretical foundation. This model and other similar approaches are reviewed and compared in [12], the method of [11] being the only one to calculate all three key operating points. On the other hand, the model proposed in [4] is theoretically justified, as it is based on the single-diode equivalent circuit. Yet, the series and shunt resistances are neglected and the slope of the $I-V$ curve at the MPP is simplistically assumed to be equal to the ratio of the OC voltage to the SC current. Similarly, the method presented in [2] is based on equations first published in [23] that do not take into account the shunt losses and require the irradiance factor of the OC voltage to be experimentally determined. This is overcome in another paper of the authors [3], where a typical value for the irradiance factor is assumed, derived from the IEC 60891 standard [22]; yet this typical value may significantly deviate from the actual one, as discussed in the following sections. A simple straightforward method to translate the entire $I-V$ curve to the actual irradiance and temperature is proposed in [5] and [6]. Still, the shunt resistance is neglected in this study, as well as in all aforementioned methods.

In this paper, a new set of simple mathematical equations to calculate the three key operating points, SC, OC and MPP, is proposed that is theoretically well-founded on the single-diode PV model. The derivation of these expressions is based on newfound relations of the irradiance and temperature coefficients with the PV module electrical characteristics available in the datasheet. This permits for the first time to calculate these parameters through other datasheet information, in contrast to the current state-of-the-art that requires experimental determination. The mathematical manipulation performed is based on the recent reformulation of the singlediode model equations to explicit form using the Lambert $W$ function [7]-[10], [26]-[29]. For this reason, new mathematical properties of the Lambert $W$ function are introduced.

The proposed equations constitute a new model that at the same time a) is in excellent agreement with the non-simplified single-diode PV model, b) is easily applied in one straightforward step, and c) relies only on datasheet information. It can be readily applied to any crystalline PV module and does not employ empirical constants. These properties render it more accurate and easy-to-use compared to other methods found in the literature, as proven by extended simulations and experimental validation performed. In fact, although there are other studies that model the irradiance and temperature effect in a similar way, the higher accuracy of the proposed method is due to the theoretically justified calculation of the respective irradiance and temperature coefficients.

The structure of the paper is as follows: the explicit reformulation of the single-diode PV model is reviewed in Section II and the proposed PV performance equations are presented in Section III. Validation through simulations and outdoor measurements is provided in Section IV and V respectively. In the Appendix, the mathematical properties of the Lambert $W$ function introduced in this paper are given.

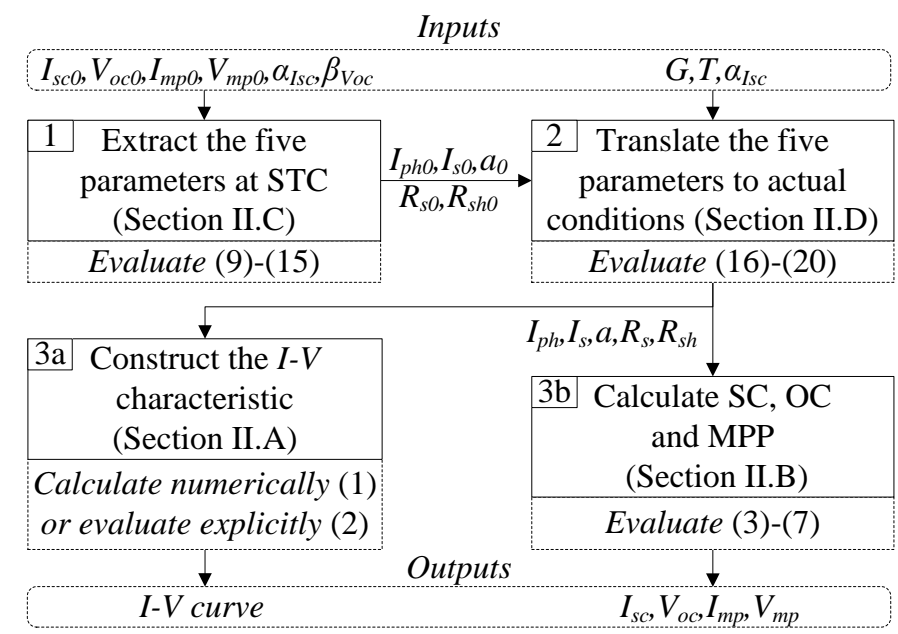

Fig. 1. Flowchart of the single-diode PV model evaluation of Section II.

\section{EXPLicit Formulation OF THE Single-DiOdE PV MODEL}

The single-diode PV model is the most widely used model in PV studies [1]-[10], [26]-[33], as it exhibits in general sufficient accuracy and reasonable complexity [2], [5]. In this section, the fundamental principles are described and an entirely explicit mathematical formulation is provided. To use this model, one has to: 1) extract the five model parameters at STC, 2) translate them to actual conditions, and 3a) construct the characteristic curve or $3 b$ ) calculate the three key operating points. These procedures are explained in detail in the following, while a flowchart that summarizes all calculation steps and input data required is given in Fig. 1.

\section{A. Equivalent Circuit}

The equivalent circuit of the PV cell is depicted in Fig. 2. It consist of a current source $I_{p h}$, a diode with a saturation current $I_{s}$ and modified diode ideality factor $a$, a series resistance $R_{s}$ and a shunt resistance $R_{s h}$. These are the five parameters of the model. This circuit is not limited only to a PV cell, but applies for any composite multi-cell system, such as a PV module, string or array, operating under uniform conditions.

The current-voltage equation of the model is given in implicit form by (1), or in equivalent explicit formulation by (2). The former expression requires iterative/numerical solution, whereas the latter can be directly evaluated by means of the Lambert $W$ function $W\{x\}$ [7]-[9], [26], [27], [31], [34].

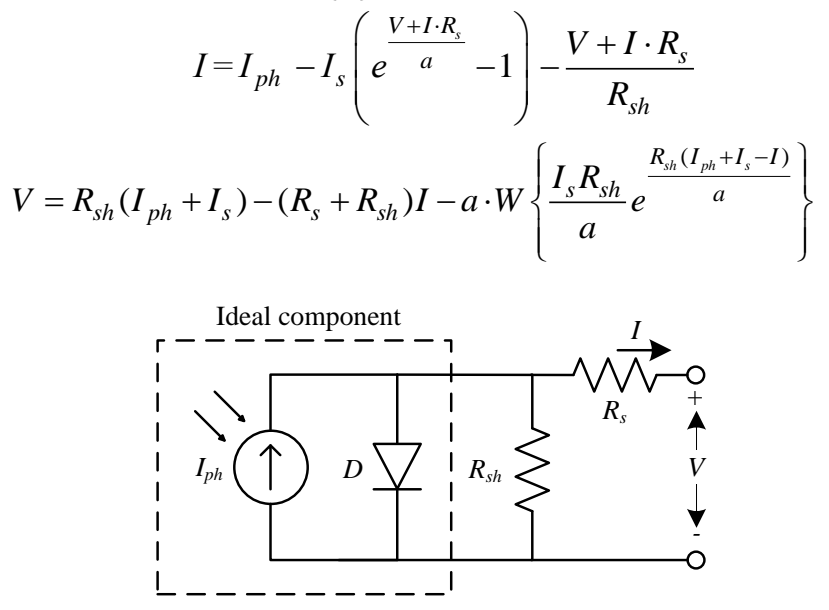

Fig. 2. PV cell single-diode electrical equivalent circuit [26]. 


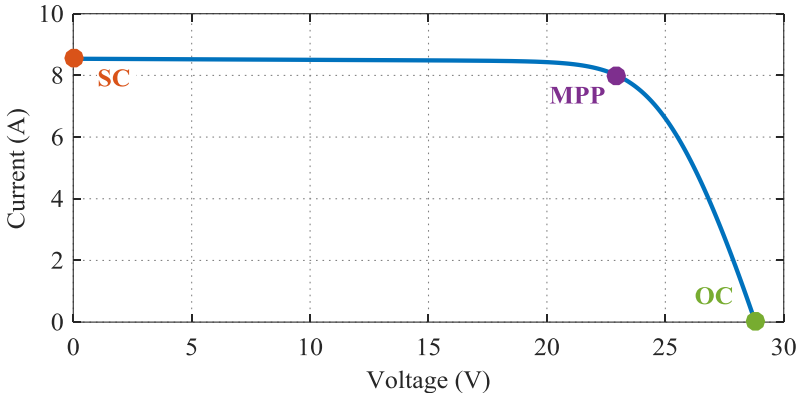

Fig. 3. Typical $I-V$ characteristic curve of a PV module at STC. The three key operating points are indicated with colored circle markers.

The $I$ - $V$ curve of a typical PV module is depicted in Fig. 3, indicating the three key operating points: SC, OC and MPP.

\section{B. Three Key Operating Points in terms of the Five Parameters}

At SC operation, the photocurrent mainly flows through the two resistances, as the diode does not conduct, essentially simplifying the circuit to a current divider [28], [31], [33]:

$$
I_{s c}=I_{p h} R_{s h} /\left(R_{s}+R_{s h}\right)
$$

At OC condition, there is no voltage drop across the series resistance and the photocurrent mainly flows through the conducting diode. Therefore, applying (1) at zero current and neglecting the two resistances yields [2], [4], [5], [7], [10] :

$$
V_{o c}=a \cdot \ln \left(I_{p h} / I_{s}\right) \Leftrightarrow I_{p h}=I_{s} e^{V_{o c} / a}
$$

The calculation of the MPP voltage and current using the five parameters is not standard in the literature. In this paper, a new set of explicit equations proposed recently by some researchers is adopted [26], [28], [29], using the notation of [26]. First, the auxiliary parameter $w$ is introduced, calculated via $I_{p h}$ and $I_{s}$, or $V_{o c}$ and $a$ if (4) is applied:

$$
w=W\left\{I_{p h} e / I_{s}\right\}=W\left\{e^{V_{o c} / a+1}\right\}
$$

Then, the MPP voltage and current are calculated through:

$$
\begin{gathered}
V_{m p}=\left(1+R_{s} / R_{s h}\right) a(w-1)-R_{s} I_{p h}(1-1 / w) \\
I_{m p}=I_{p h}(1-1 / w)-a(w-1) / R_{s h} \\
V_{m p}=a(w-1)-R_{s} I_{m p}
\end{gathered}
$$

The derivation of these equations is based on the observation that the output power of the entire cell and its ideal component (Fig. 2) practically become maximum at the same time [26].

\section{Extraction of the Five Reference Parameters at STC}

Few straightforward methods (non-iterative algorithms) to directly evaluate the five parameters are found in the literature [4], [5], [7], [10], [32], [33]. This paper adopts the approach of [7], as it is based on the non-simplified single-diode model and relies only on datasheet information. Therein, a new coefficient $\delta_{0}$ is introduced defined as the ratio of the parameter $a_{0}$ over $V_{o c 0}$ (values at STC), given by:

$$
\delta_{0}=a_{0} / V_{o c 0}=\left(1-\beta_{V o c} T_{0}\right) /\left(50.1-a_{I s c} T_{0}\right)
$$

where $T_{0}=298.15 \mathrm{~K}$ is the STC temperature and $\beta_{V o c}, \alpha_{I s c}$ are the normalized temperature coefficients of the OC voltage and SC current (e.g. $\beta_{V o c}=-0.0034 \mathrm{~K}^{-1}, \alpha_{I s c}=+0.0006 \mathrm{~K}^{-1}$ ). As discussed in Section III, $\delta_{0}$ stands for the irradiance factor of the OC voltage, while it has a typical value of 0.04 [7]. The auxiliary parameter $w_{0}$ is then expressed at STC as a function of the coefficient $\delta_{0}$ substituting (9) into (5) for STC:

$$
w_{0}=W\left\{I_{p h 0} e / I_{s 0}\right\}=W\left\{e^{V_{o c 0} / a_{0}+1}\right\}=W\left\{e^{1 / \delta_{0}+1}\right\}
$$

Having determined $\delta_{0}$ and $w_{0}$, the five reference parameters $I_{p h o}, I_{s 0}, a_{0}, R_{s 0}$ and $R_{s h o}$ are explicitly calculated by sequential evaluation of (11)-(15):

$$
\begin{gathered}
a_{0}=\delta_{0} V_{o c 0} \\
R_{s 0}=\left[a_{0}\left(w_{0}-1\right)-V_{m p 0}\right] / \mathrm{I}_{m p 0} \\
R_{s h 0}=a_{0}\left(w_{0}-1\right) /\left[I_{s c 0}\left(1-1 / w_{0}\right)-\mathrm{I}_{m p 0}\right] \\
I_{p h 0}=\left(1+R_{s 0} / R_{s h 0}\right) I_{s c 0} \\
I_{s 0}=I_{p h 0} e^{-1 / \delta_{0}}
\end{gathered}
$$

where $I_{s c 0}, V_{o c 0}, I_{m p 0}$ and $V_{m p 0}$ correspond to the three key operating points at STC, always given in the module datasheet.

\section{Translation of the Five Parameters to any Conditions}

In order to translate the five reference parameters to the actual irradiance $G$ and temperature $T$, the translation equations proposed in [1] are used, as manipulated in [7] and [30]:

$$
\begin{gathered}
I_{p h}=I_{p h 0} G\left(1+\alpha_{I s c} \Delta T\right) \\
I_{s}=I_{s 0} \lambda T^{3} e^{47.1(1-1 / \lambda T)} \\
a=a_{0} \lambda T \\
R_{s}=R_{s 0} \\
R_{s h}=R_{s h 0} / G
\end{gathered}
$$

where the temperature deviation from the STC value $T_{0}$ is expressed as a difference $\Delta T=T-T_{0}$ or a ratio $\lambda T=T / T_{0}$ (values in Kelvin). Furthermore, the auxiliary parameter $w$ is translated to $G$ and $T$ according to (details in the Appendix):

$$
w=w_{0}+\frac{1}{1+\delta_{0}}\left(\frac{\beta_{V o c}-1 / T_{0}}{\delta_{0}} \frac{\Delta T}{\lambda T}+\ln G\right)
$$

\section{Proposed PV Performance Equations}

As discussed in the Introduction, there is a need for direct evaluation of the three key operating points at any conditions in a single straightforward step. The expressions (3)-(7) are not the best option for this, as they are functions of the five parameters and require sequential application of almost all equations given in Section II via a three-step calculation procedure (see flowchart of Fig. 1). In the following, it is shown that these equations can be symbolically combined to derive simple SC, OC and MPP expressions that are functions directly of the operating conditions $G$ and $T\left(\Delta T=T-T_{0}, \lambda T=T / T_{0}\right)$. The required input data are only the datasheet electrical characteristics: $I_{s c 0}, V_{o c 0}, I_{m p 0}, V_{m p 0}, \alpha_{I s c}$ and $\beta_{V o c}$ :

$$
\begin{gathered}
I_{s c}=G \cdot I_{s c 0}\left(1+a_{I s c} \Delta T\right) \\
V_{o c}=V_{o c 0}\left(1+\delta_{0} \lambda T \ln G+\beta_{V o c} \Delta T\right) \\
I_{m p}=G \cdot I_{m p 0}\left(1+a_{I m p} \Delta T\right) \\
V_{m p}=V_{m p 0}\left[1+\varepsilon_{0} \lambda T \ln G+\varepsilon_{1}(1-G)+\beta_{V m p} \Delta T\right]
\end{gathered}
$$

where $\delta_{0}$ is the irradiance factor of the OC voltage calculated via (9), $\varepsilon_{0}, \varepsilon_{1}$ are the irradiance factors of the MPP voltage: 


$$
\varepsilon_{0}=\frac{\delta_{0}}{1+\delta_{0}} \frac{V_{o c 0}}{V_{m p 0}}, \quad \varepsilon_{1}=\delta_{0}\left(w_{0}-1\right) \frac{V_{o c 0}}{V_{m p 0}}-1
$$

and $\alpha_{I m p}, \beta_{V m p}$ are the normalized temperature coefficients of the MPP current and voltage:

$$
\begin{gathered}
a_{I m p}=a_{I s c}+\left(\beta_{V o c}-1 / T_{0}\right) /\left(w_{0}-1\right) \\
\beta_{V m p}=\frac{V_{o c 0}}{V_{m p 0}}\left[\frac{\beta_{V o c}}{1+\delta_{0}}+\frac{\delta_{0}\left(w_{0}-1\right)-1 /\left(1+\delta_{0}\right)}{T_{0}}\right]
\end{gathered}
$$

The parameter $w_{0}$ is calculated through (10) using $\delta_{0}$. If the three key operating points are determined via (22)-(25), the maximum power $P_{m p}$ and the fill factor $F F$ are readily derived:

$$
P_{m p}=V_{m p} I_{m p}, \quad F F=\left(V_{m p} I_{m p}\right) /\left(V_{o c} I_{s c}\right)
$$

Although the above equations refer to a single PV module, they apply for any larger PV system with proper adjustment (multiply $I_{s c}, I_{m p}$ by the number of parallel-connected modules and $V_{o c}, V_{m p}$ by the number of series-connected modules) [35], [36]. The derivation steps of these equation are given below.

\section{A. Short Circuit (SC) Current $-I_{s c}$}

The SC current $I_{s c}$ is generally considered to vary proportionally with the irradiance $G$ and linearly with the temperature $T$ [2]-[6], [13]-[19], [21]-[23]. The adopted translation equation is given by (22), where the temperature coefficient $\alpha_{\text {Isc }}$ is always provided in the module datasheet. There are only few studies in the literature that deviate from this approach: an exponential irradiance dependence is assumed in [24], while in [11], [12] the temperature effect is ignored.

Regarding the temperature coefficient $\alpha_{I s c}$, it may be used in either absolute $(\mathrm{A} / \mathrm{K})$ [18], [19], [21], [22] or normalized $(1 / \mathrm{K})$ [2]-[6], [13]-[17], [23] form. The latter approach, i.e. (22), is preferred in the literature, as the normalized coefficient does not considerably differ among PV modules of the same technology, which facilitates comparison [13]. All temperature coefficients $\alpha_{I s c}, \alpha_{I m p}, \beta_{V o c}$ and $\beta_{V m p}$ are used in normalized form in this paper.

\section{B. Open Circuit (OC) Voltage - $V_{o c}$}

Most of the relevant studies consider the OC voltage $V_{o c}$ to be affected in a logarithmic way by $G$ and in a linear way by $T$ [2], [5], [13]-[19], [21]-[23]. Only a few methods neglect the irradiance effect [11], [12]. The proposed (23) is derived if the leftmost version of (4) is employed and (16)-(18) are applied for the translation of $I_{p h}, I_{s}$ and $a$ :

$$
V_{o c}=a_{0} \lambda T \ln G+\underbrace{a_{0} \lambda T \ln \left[\frac{I_{p h 0}\left(1+\alpha_{I s c} \Delta T\right)}{I_{s 0} \lambda T^{3} e^{47.1(1-1 / \lambda T)}}\right]}_{V_{o c}\left(G_{0}=1, T\right)=V_{o c 0}\left(1+\beta_{v o c} \Delta T\right)}
$$

The last term of (30) corresponds to the OC voltage at nominal irradiance $G_{0}=1$ and arbitrary temperature $T$, which may be modeled through the coefficient $\beta_{V o c}$ as: $V_{o c 0}\left(1+\beta_{V o c} \Delta T\right)$. Substituting this equation into (30) and applying (11) for $a_{0}$

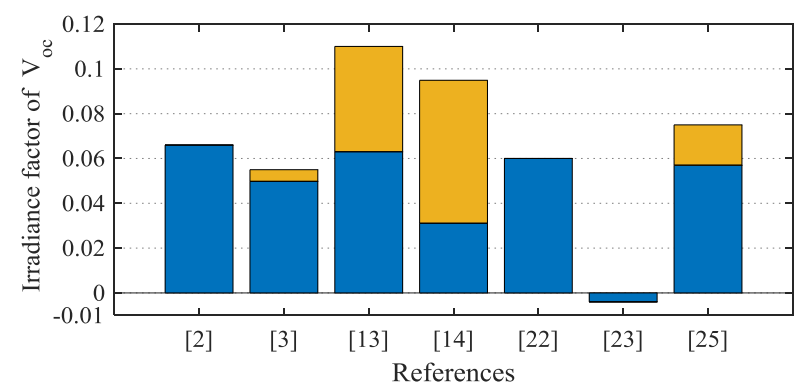

Fig. 4. Irradiance factor of $V_{o c}$ reported in references: [2], [3], [13], [14], [22], [23], [25]. The orange bars indicate range of values.

leads to (23). The temperature coefficient $\beta_{V o c}$ is provided in the module datasheet and is considered constant and unaffected by the irradiance for non-concentrator flat-plate modules [21].

The middle term in the parenthesis of (23) reflects the logarithmic irradiance effect, found in the abovementioned references in equivalent formulation (some of them neglect the temperature effect in this term):

$$
\delta_{0} V_{o c 0} \lambda T \ln G=a_{0} \frac{T}{T_{0}} \ln G=N_{s} n \frac{k \cdot T}{q} \ln G=N_{s} n V_{T} \ln G
$$

where $N_{s}$ is the number of series-connected cells, $n$ the diode ideality factor, $k$ the Boltzmann constant, $q$ the electron charge and $V_{T}$ the thermal voltage. However, all of these approaches require either the irradiance factor $\delta_{0}$ or the diode ideality factor $n$ as input data, none of which are normally given in the module datasheet. This leads to additional measurements or the adoption of typical values, such as the one proposed in the IEC 60891 standard: $\delta_{0}=0.06$ [22]. Yet, the selection of the appropriate value for the irradiance factor is not a trivial task, as it depends on the technology and structural characteristics of the PV modules. In the literature, the irradiance factor varies significantly from slightly negative up to 0.11 , as shown in Fig. 4. On the other hand, the proposed approach to calculate the irradiance factor $\delta_{0}$ through (9) directly from datasheet information, permits better adaptation to the study-case system. This is the first method to provide this flexibility.

\section{Maximum Power Point (MPP) Current - Imp}

The translation equation of the MPP current $I_{m p}$ is not standard in the literature. Most studies consider a proportional irradiance effect [2], [13]-[19], [22], [23] and others quadratic irradiance dependence [21], while the temperature effect remains somewhat unclear. In this paper, first the translation equations of the parameters $I_{p h}, a, R_{s h}$ and $w$ (16), (18), (20), (21) are substituted into (7), resulting after some manipulation in (24), where $\alpha_{\operatorname{Imp}}$ is provided by the huge expression (32) at the bottom of the page. Clearly, this equation is too complicated for practical applications, thus it is simplified to (details in the Appendix):

$$
a_{I m p}=a_{I s c}+\frac{\beta_{V o c}-1 / T_{0}}{w_{0}-1} \frac{1+\alpha_{I s c} \Delta T}{1+\beta_{V o c} \Delta T}
$$

$$
a_{I m p}=\frac{I_{s c 0}}{I_{m p 0}}\left[a_{I s c}+\frac{1}{w_{0} \Delta T}-\frac{1+a_{I s c} \Delta T}{w_{0} \Delta T+\frac{1}{1+\delta_{0}}\left(\frac{\beta_{V o c}-1 / T_{0}}{\delta_{0}} \frac{\Delta T}{\lambda T}+\ln G\right) \Delta T}\right]-\left(\frac{I_{s c 0}}{I_{m p 0} w_{0}} \frac{1}{w_{0}-1}\right)\left[\frac{w_{0}-1}{T_{0}}+\frac{1}{1+\delta_{0}}\left(\frac{\beta_{V o c}-1 / T_{0}}{\delta_{0}} \frac{\Delta T}{\lambda T}+\ln G\right) \frac{\lambda T}{\Delta T}\right]
$$


According to (33), $\alpha_{I m p}$ does not coincide with $\alpha_{I s c}$ nor is it a constant: it is a rational function of the temperature $T$ having $\alpha_{I s c}$ as a dominant term. Yet, the temperature dependence on (33) is found to be very limited, thus permitting the adoption of a typical value for $\alpha_{I m p}$, equal to $\alpha_{I m p}(\Delta T=0)$ at nominal temperature $\left(T=T_{0}\right)$. The final expression for $\alpha_{\operatorname{lmp}}$ is (27), where the temperature coefficient is a constant instead of a function.

In the literature, it is often simplistically assumed that $\alpha_{I m p}=\alpha_{I s c}$ [2], [13]-[17], [22], [23]. Yet, the tests of the SANDIA laboratories have verified that $\alpha_{I m p}$ practically differs from $\alpha_{I s c}$, thus imposing separate experimental determination [18], [19], [21]. The proposed (27) permits calculation of $\alpha_{I m p}$ directly from the datasheet, leading to greater accuracy compared to the assumption that $\alpha_{I m p}=\alpha_{I s c}$ or $\alpha_{I m p}=0$, as discussed in Section IV.

\section{Maximum Power Point (MPP) Voltage $-V_{m p}$}

As for the MPP voltage $V_{m p}$, there is no commonly accepted formula. The irradiance effect is modelled logarithmically in [13]-[17], in a combined linear-logarithmic way in [2], [22], [23], or as a binomial of logarithms in [18], [19], [21]. In this paper, it is found that the irradiance dependence on $V_{m p}$ is linear-logarithmic, as envisaged in the IEC 60891 standard [22]. In particular, if the translation equations of the parameters $a, R_{s}$ and $w(18),(19),(21)$ are substituted into (8), and (24) is applied for $I_{m p}$, the following expression is derived:

$$
\begin{aligned}
V_{m p}= & a_{0} \lambda T\left[w_{0}+\frac{1}{1+\delta_{0}}\left(\frac{\beta_{V o c}-1 / T_{0}}{\delta_{0}} \frac{\Delta T}{\lambda T}+\ln G\right)-1\right] \\
& +R_{s 0} G \cdot I_{m p 0}\left(1+\alpha_{m m p} \Delta T\right)
\end{aligned}
$$

In (34), the temperature effect on the current is ignored since it is negligible compared to the temperature effect on the voltage. Thereafter, writing (8) at STC and substituting into (34) for the term $R_{s 0} I_{m p 0}$ leads to (25), where $\varepsilon_{0}$ and $\varepsilon_{l}$ are the irradiance factors given by (26), and $\beta_{V m p}$ is the normalized temperature coefficient provided by (28).

$\varepsilon_{0}$ is the coefficient of the logarithmic irradiance term in $V_{m p}$ equation (25), in the same way that $\delta_{0}$ is the coefficient of the logarithmic irradiance term in $V_{o c}$ equation (23). The values of the two constants are very similar ( $\varepsilon_{0}$ is always slightly greater than $\delta_{0}$ ). $\varepsilon_{l}$ is the coefficient of the linear irradiance term, which is in general 1.5-3 times greater than $\delta_{0}$. In [13]-[17], only one irradiance factor is considered for both $V_{o c}$ and $V_{m p}$ equations, whereas two different coefficients are used in [2], [18], [19], [21]-[23] for the $V_{m p}$ equation; in either case, experimental determination is required.

As for the temperature coefficient $\beta_{V m p}$, evaluation of (28) reveals that: $\beta_{V m p} V_{m p} \approx \beta_{V o c} V_{o c}$, i.e. the temperature coefficients of the OC and MPP voltage are similar in absolute form. This is why the same (absolute) temperature coefficient is used for both cases in [13]-[17]. Yet, again the experimental tests of the SANDIA laboratories have concluded that these two coefficients are in general different (even slightly) and should be determined separately. This is achieved via additional measurements in all relevant studies [2], [13]-[19], [21]-[23]. The proposed expressions for the irradiance and temperature coefficients (26) and (28) are the first equations to permit direct evaluation of these constants directly from the datasheet.

\section{VALIDATION THROUGH SIMULATIONS IN MATLAB}

To validate the proposed PV performance model, simulations are performed in MATLAB on 20 mono- and poly- crystalline PV modules derived from [7]. Every PV module is simulated at several scenarios corresponding to different irradiance (from 200 to $1000 \mathrm{~W} / \mathrm{m}^{2}$ with a step of $50 \mathrm{~W} / \mathrm{m}^{2}$ ) and temperature (from -5 to $65^{\circ} \mathrm{C}$ with a step of $5{ }^{\circ} \mathrm{C}$ ), resulting in 255 scenarios per module (5100 scenarios in total).

First, the coefficients expressions are studied, and then the PV performance equations are applied and compared to other methods found in the literature. The single-diode PV model is adopted as a reference, in the way presented by De Soto in [1].

\section{A. Validation of the Coefficients Equations}

In order to evaluate the accuracy of (9), (26)-(28) in estimating the irradiance factors and temperature coefficients, the reference/actual values of these constants need to be determined. This is accomplished by performing surface fitting [37]. First, every PV module is simulated by the De Soto model for all 255 scenarios to acquire the $I-V$ curve and $V_{o c}, I_{m p}, V_{m p}$ over the entire range of irradiance and temperature. Then, surface fitting is performed for $V_{o c}$ applying (23) to the simulated data, in order to determine the irradiance coefficient that achieves optimal fitting. This is the actual value of $\delta_{0}$. Thereafter, the same procedure is repeated for $I_{m p}$ and $V_{m p}$, applying (24) and (25), to determine the actual values of $\alpha_{I m p}$ and $\varepsilon_{0}, \varepsilon_{1}, \beta_{V m p}$ respectively.

In Fig. 5(a), the extracted/actual (continuous lines) and estimated (cross markers) irradiance factors are depicted. As shown, $\delta_{0}$ and $\varepsilon_{0}$ present small deviation around 0.04 and 0.05 respectively, whereas $\varepsilon_{l}$ varies significantly between 0.03 and 0.12 for the study-case modules. The temperature coefficients are illustrated in Fig. 5(b): $\beta_{V m p}$ varies around $-0.004 \mathrm{~K}^{-1}$, which is approximately $30 \%$ greater in magnitude than $\beta_{V o c}$, while $\alpha_{I m p}$ takes very small values; yet, it seems that $\alpha_{\operatorname{Imp}}$ should not be

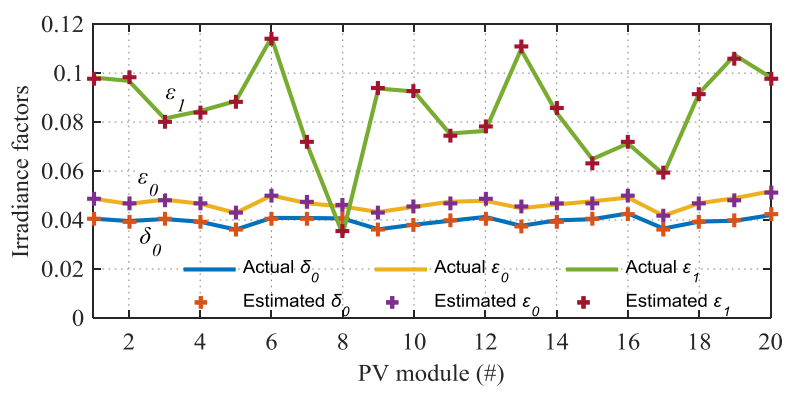

(a)

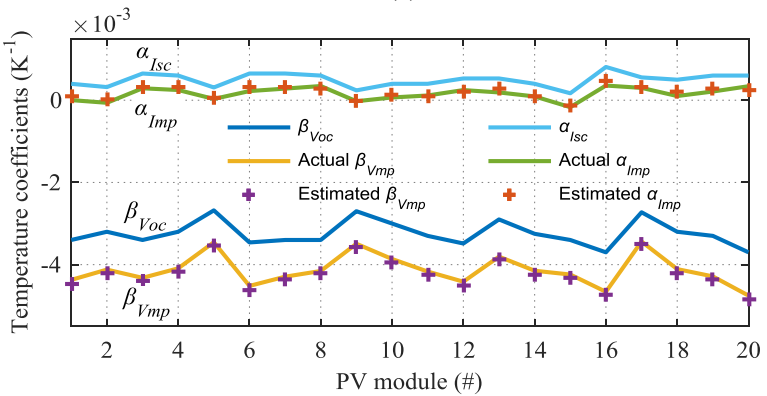

(b)

Fig. 5. (a) Irradiance factors, and (b) temperature coefficients for 20 crystalline PV modules, extracted via surface fitting (actual values) and through the proposed (9), (26)-(28) (estimated values). 
TABLE I

MEAN IRRADIANCE AND TEMPERATURE COEFFICIENTS FOR 20 PV MOdULES

\begin{tabular}{cccrrrr}
\hline $\boldsymbol{\delta}_{\boldsymbol{\theta}}$ & $\boldsymbol{\varepsilon}_{\boldsymbol{\theta}}$ & $\boldsymbol{\varepsilon}_{\boldsymbol{l}}$ & $\boldsymbol{\alpha}_{\boldsymbol{I s c}}\left(\mathrm{K}^{-1}\right)$ & $\boldsymbol{\alpha}_{\boldsymbol{I m p}}\left(\mathrm{K}^{-1}\right)$ & $\boldsymbol{\beta}_{\text {Voc }}\left(\mathrm{K}^{-1}\right)$ & $\boldsymbol{\beta}_{\text {Vmp }}\left(\mathrm{K}^{-1}\right)$ \\
\hline 0.0396 & 0.0468 & 0.0846 & 0.00049 & 0.00016 & -0.0032 & -0.0041 \\
\hline
\end{tabular}

considered equal to neither $\alpha_{I s c}$ nor zero. In all cases, excellent convergence is observed between the estimated and actual values, thus validating the respective (9), (26)-(28). The mean values of these coefficients for the 20 study-case PV modules are given in Table I for comparison reasons; yet, the simulation results indicate that these values should not be used as typical coefficients, as there is significant variation from module to module, especially for different technologies.

\section{B. Validation of the PV Performance Equations}

The proposed performance model is evaluated in all 5100 simulation scenarios, recording the accuracy in calculating the six common energy yield indices: $I_{s c}, V_{o c}, I_{m p}, V_{m p}, P_{m p}$ and $F F$. Four other PV models found in the literature are applied and compared: Araujo [11], [12], Saloux [4], Ding [2] and Cristaldi [5], [6]. As discussed in the introduction, these models permit direct estimation of the aforementioned parameters, while they rely solely on datasheet information, as the proposed equations.

As a reference, the well-established De Soto model [1] is used: first, the five reference parameters are extracted at STC for each PV module; then, these parameters are translated to the irradiance and temperature of each simulation scenario and the $I-V$ curve is constructed; thereafter, the SC, OC and MPP operating points are recorded to determine the actual values of the performance indices; finally, the five performance models are applied and the estimation accuracy is evaluated.

In Fig. 6, the error distribution of the MPP power is depicted, as it is the most representative energy yield index. Each value corresponds to the rms of the errors calculated for the 20 studycase PV modules. The recorded accuracy proves to be very good over the entire range of the irradiance and temperature, exhibiting a global maximum error of $0.65 \%$ and an overall rms error of $0.33 \%$. The lowest estimation accuracy is observed at the highest values of the irradiance and temperature, when the equations (6)-(8) and (11)-(15) integrated into the Proposed model present their greatest errors [7], [26].

The rms error in calculating the six energy yield indices is shown in Table II (collectively for the 20 PV modules - all 5100 scenarios). $I_{s c}$ estimation is exceptional in all but the Araujo method, which ignores the temperature effect. For the $V_{o c}$, although the Ding and Cristaldi models adopt a very similar equation to (23), the irradiance factors selected are not entirely

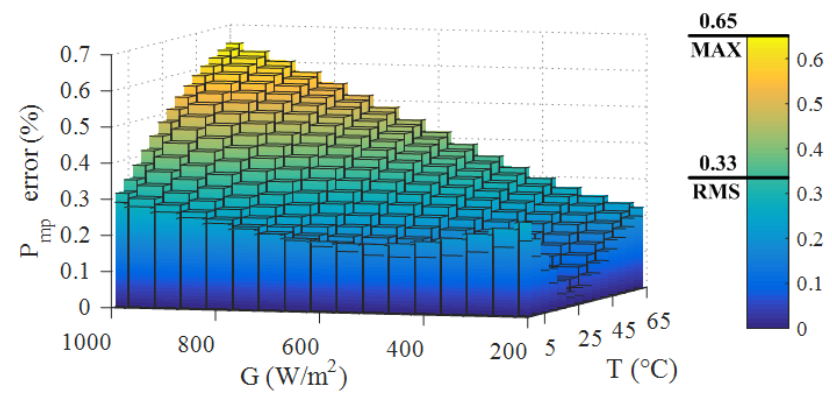

Fig. 6. Error distribution in estimating the MPP power over the entire range of irradiance and temperature, applying the Proposed equations. For each irradiance/temperature scenario, the rms error of all 20 modules is plotted.
TABLE II

RMS ERRor IN ESTIMATING THE PERFORMANCE INDICES BY FIVE PV MODELS USING SIMULATIONS ON 20 PV MODULES

\begin{tabular}{|c|c|c|c|c|c|c|c|}
\hline Method & $I_{s c}$ & $V_{o c}$ & $I_{m p}$ & $V_{m p}$ & $P_{m p}$ & $F F$ & Overall \\
\hline Proposed & 0.1 & 0.1 & 0.3 & 0.2 & 0.3 & 0.3 & 0.23 \\
\hline Araujo & 1.2 & 3.4 & 6.6 & 6.7 & 2.2 & 4.1 & 4.53 \\
\hline Saloux & 0.1 & 2.5 & 1.5 & 5.9 & 7.0 & 4.6 & 4.35 \\
\hline Ding & 0.1 & 1.6 & 0.8 & 3.3 & 3.6 & 2.1 & 2.29 \\
\hline Cristaldi & 0.1 & 1.3 & 1.2 & 3.2 & 3.7 & 2.3 & 2.32 \\
\hline
\end{tabular}

appropriate, resulting in small errors. The other two approaches, Araujo and Saloux, exhibit even lower accuracy due to the assumptions made. The estimation of $I_{m p}$ by the Ding method proves very satisfactory, since the same equation as (24) is adopted; yet, $\alpha_{I m p}$ is simplistically assumed to be equal to $\alpha_{I S c}$, resulting in slightly greater error than the Proposed approach. As for $V_{m p}$, it seems that it is a very difficult-to-determine performance index for all but the Proposed model, mainly due to the complicated irradiance effect. As discussed in Section III.D, there is no commonly accepted modelling formula in the literature; nevertheless, the high accuracy brought by (25) strengthens the linear-logarithmic approach. Finally, $P_{m p}$ and $F F$ parameters are calculated indirectly based on the previous four performance indices, thus the resulting accuracy depends on the errors brought by the latter parameters. In any case, the Proposed approach exhibits the best performance in all energy yield indices among the five PV models.

It is worth noting, that although the form of the SC, OC and MPP equations (22)-(25) is not new in the literature, it is the newly-introduced coefficients expressions (9), (26)-(28) that contribute to the higher accuracy. However, this superiority essentially indicates excellent agreement with the single-diode $P V$ model, rather than best performance in every real-life scenario, as discussed in detail in the following section.

\section{VALIDATION THROUGH OUTDOOR MEASUREMENTS}

Experimental measurements were performed on 5 commercial crystalline PV modules to further validate and compare the five PV models in outdoor conditions. The $I-V$ curve and operating conditions were recorded by the Amprobe Solar-4000 analyzer (Fig. 7). The irradiance sensor is equipped with one mono- and one poly- crystalline cell and is mounted on the module frame to get the correct inclination. This way an accurate measurement of the effective irradiance is acquired. The temperature is measured at several parts of the PV module

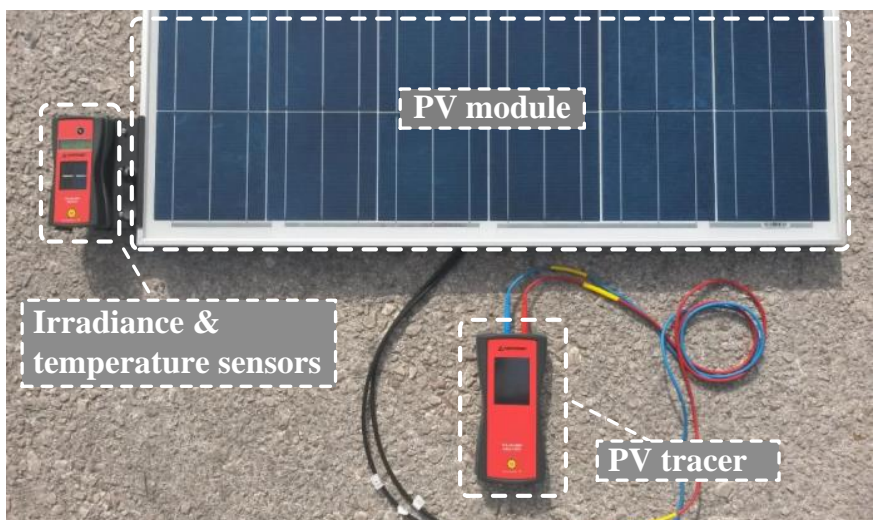

Fig. 7. Measurement equipment (Amprobe Solar-4000 analyzer) used for the experimental validation of the five PV models. 


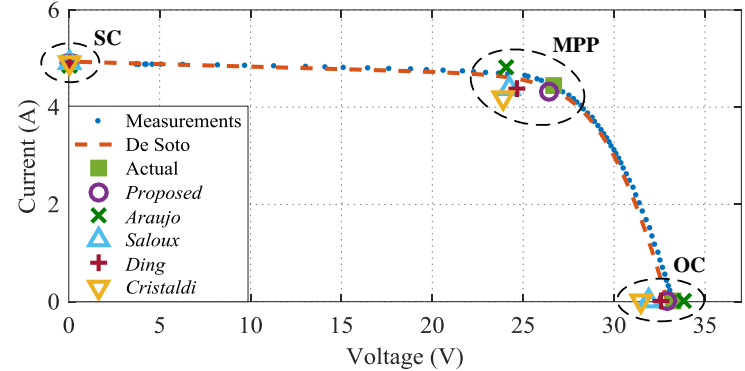

Fig. 8. Measured $I-V$ curve indicating the actual and estimated SC, OC and MPP operating points using the five PV models (Perllight PLM-250P-60 @ $\left.570 \mathrm{~W} / \mathrm{m}^{2}-54{ }^{\circ} \mathrm{C}\right)$.

in a contactless manner using an infrared sensor immediately after the $I-V$ curve has been captured: the output cell temperature is the average of these samples. The manufacturer guarantees a measurement error up to $\pm 5 \%$.

Each module was measured at three different scenarios of irradiance and temperature, leading to 15 sets of measurements in total. The irradiance ranges from 440 to $965 \mathrm{~W} / \mathrm{m}^{2}$ and the temperature from 31 to $59{ }^{\circ} \mathrm{C}$. To facilitate comparison, these measurements are classified into two categories: High irradiance for values greater than $700 \mathrm{~W} / \mathrm{m}^{2}$ and Low irradiance for the rest. The procedure followed is quite simple: at each scenario, the $I-V$ curve was recorded and the three key operating points were located; then, the five PV models were applied for the measured irradiance/temperature and the six performance indices were calculated. In the following, the results of this comparison are presented.

An indicative measured $I-V$ curve (blue dots) is illustrated in Fig. 8, along with the respective simulated characteristic derived by the De Soto model (red dashed line). The actual and estimated three key operating points are depicted with colored markers. As expected, the estimated points by the Proposed model (purple circle markers) coincide with the simulated $D e$ Soto characteristic. The SC current is sufficiently estimated via all methods, while the OC voltage is underestimated by the Saloux and Cristaldi approaches. At the MPP, only the Proposed model manages to accurately determine both the voltage and current. All other methods underestimate $V_{m p}$, while $I_{m p}$ is sufficiently calculated by the Saloux, Ding and Cristaldi methods, and is overestimated by the Araujo approach. Nevertheless, it is worth noting that the latter model provides a fairly accurate estimation of $P_{m p}$.

These observations are further confirmed in Table III, where the $P_{m p}$ estimation error is shown for all measured scenarios. It is worth noting that the lowest error (bold font) is brought by a different method in every case. Nevertheless, if one focuses on the overall rms and global maximum errors, it is evident that the Proposed and Araujo models exhibit the most favorable performance. The other three methods result in high estimation errors in some cases, which implies reduced reliability for practical applications.

The complete picture on the overall effectiveness of the five PV models is provided in Table IV, where the rms error in calculating all six performance indices is shown separately for the two irradiance subsets. The results in the High irradiance cases indicate that the Proposed method performs most
TABLE III

ESTIMATION MPP POWER ERROR BY FIVE PV MODELS USING OUTDOOR MEASUREMENTS ON 5 PV MODULES

\begin{tabular}{|c|c|c|c|c|c|}
\hline \multirow{2}{*}{$\begin{array}{c}\text { PV module } \\
\text { Irradiance - Temperature }\end{array}$} & \multicolumn{5}{|c|}{$P_{m p}(\%)$ estimation error } \\
\hline & Proposed & Araujo & Saloux & Ding & Cristaldi \\
\hline \multicolumn{6}{|l|}{ Conergy PowerPlus 190PC } \\
\hline $965 \mathrm{~W} / \mathrm{m}^{2}-44^{\circ} \mathrm{C}$ & 2.4 & 3.5 & 3.3 & 3.2 & 2.1 \\
\hline $775 \mathrm{~W} / \mathrm{m}^{2}-40{ }^{\circ} \mathrm{C}$ & 2.8 & 3.0 & 0.9 & 2.4 & 1.9 \\
\hline $625 \mathrm{~W} / \mathrm{m}^{2}-36^{\circ} \mathrm{C}$ & 2.8 & 2.5 & -1.4 & 1.5 & 1.4 \\
\hline \multicolumn{6}{|l|}{ Perllight PLM-250P-60 } \\
\hline $960 \mathrm{~W} / \mathrm{m} 2-44^{\circ} \mathrm{C}$ & -2.4 & -1.2 & 2.3 & -2.1 & -4.1 \\
\hline $800 \mathrm{~W} / \mathrm{m} 2-50^{\circ} \mathrm{C}$ & -1.8 & -0.5 & -4.2 & -3.7 & -7.5 \\
\hline $570 \mathrm{~W} / \mathrm{m} 2-54{ }^{\circ} \mathrm{C}$ & -3.2 & -2.0 & -10.1 & -9.0 & -15.4 \\
\hline \multicolumn{6}{|l|}{ Solea SM 190} \\
\hline $910 \mathrm{~W} / \mathrm{m} 2-31^{\circ} \mathrm{C}$ & 1.2 & 1.3 & 0.4 & 0.9 & 0.5 \\
\hline $730 \mathrm{~W} / \mathrm{m} 2-37{ }^{\circ} \mathrm{C}$ & 1.0 & 1.2 & -2.0 & -0.3 & -1.1 \\
\hline $550 \mathrm{~W} / \mathrm{m} 2-40^{\circ} \mathrm{C}$ & 2.8 & 3.1 & -3.0 & 0.1 & -0.9 \\
\hline \multicolumn{6}{|l|}{ Day4 Energy 60MC-I } \\
\hline $895 \mathrm{~W} / \mathrm{m} 2-53{ }^{\circ} \mathrm{C}$ & 3.4 & 5.3 & 3.8 & 4.3 & 2.9 \\
\hline $740 \mathrm{~W} / \mathrm{m} 2-47^{\circ} \mathrm{C}$ & 3.6 & 4.0 & 0.9 & 3.3 & 2.5 \\
\hline $525 \mathrm{~W} / \mathrm{m} 2-42{ }^{\circ} \mathrm{C}$ & 3.0 & 2.2 & -3.8 & 1.1 & 1.1 \\
\hline \multicolumn{6}{|l|}{ Yingli YL-165 } \\
\hline $925 \mathrm{~W} / \mathrm{m} 2-59^{\circ} \mathrm{C}$ & 0.8 & 4.0 & 1.6 & 1.8 & -1.3 \\
\hline $575 \mathrm{~W} / \mathrm{m} 2-50{ }^{\circ} \mathrm{C}$ & 0.7 & 1.9 & -5.8 & -2.7 & -6.7 \\
\hline $440 \mathrm{~W} / \mathrm{m} 2-46^{\circ} \mathrm{C}$ & -0.2 & 0.7 & -9.8 & -5.5 & -9.8 \\
\hline OVERALL RMS & 2.4 & 2.8 & 4.6 & 3.6 & 5.7 \\
\hline GLOBAL MAXIMUM & 3.6 & 5.3 & -10.1 & -9.0 & 15.4 \\
\hline
\end{tabular}

favorably overall, bringing the lowest or near-lowest errors in all performance indices. When comparing with the Low irradiance results, the recorded errors prove to be slightly higher, since all models exhibit lower accuracy at low irradiance. In these cases, the Araujo model provides a fairly good estimation of $P_{m p}$ and $F F$ despite the large $I_{m p}$ and $V_{m p}$ errors, as these deviations seem to counterbalance each other. However, in these cases too, the recorded deviation by the Proposed method is very close to the apparent lowest error. Furthermore, when focusing on the overall rms errors, the Proposed model provides almost the same level of accuracy regardless of the irradiance level, in contrast to the other models. This implies increased estimation accuracy and reliability in the common everyday scenarios.

In fact, the main conclusion from this experimental investigation is that the Proposed equations are as accurate as the single-diode PV model in real-life conditions. This verifies the strong theoretical background that does not allow for high errors, regardless of the PV module or operating conditions. Consequently, this is probably the most favorable option for a reliable overall performance estimation.

TABLE IV

RMS ERROR IN ESTIMATING ALL SIX PERFORMANCE INDICES BY FIVE PV MODELS USING OUTDOOR MEASUREMENTS ON 5 PV MODULES

\begin{tabular}{|c|c|c|c|c|c|c|c|}
\hline Method & $I_{s c}$ & $V_{o c}$ & $I_{m p}$ & $V_{m p}$ & $P_{m p}$ & $F F$ & Overall \\
\hline \multicolumn{8}{|c|}{ High irradiance $\left(\geq 700 \mathrm{~W} / \mathrm{m}^{2}\right)$} \\
\hline Proposed & 0.7 & 0.2 & 2.1 & 1.9 & 2.2 & 1.7 & 1.66 \\
\hline Araujo & 0.9 & 1.6 & 7.3 & 5.2 & 3.0 & 2.8 & 4.11 \\
\hline Saloux & 0.7 & 1.3 & 1.3 & 3.0 & 3.3 & 2.6 & 2.27 \\
\hline Ding & 0.7 & 0.7 & 1.8 & 1.7 & 2.6 & 2.4 & 1.80 \\
\hline Cristaldi & 0.7 & 1.2 & 2.7 & 2.1 & 3.9 & 3.1 & 2.54 \\
\hline \multicolumn{8}{|c|}{ Low irradiance $\left(<700 \mathrm{~W} / \mathrm{m}^{2}\right)$} \\
\hline Proposed & 0.7 & 0.5 & 1.4 & 1.5 & 2.6 & 2.3 & 1.68 \\
\hline Araujo & 0.9 & 2.3 & 7.7 & 6.1 & 2.4 & 2.0 & 4.32 \\
\hline Saloux & 0.7 & 2.5 & 0.8 & 5.2 & 6.0 & 3.9 & 3.80 \\
\hline Ding & 0.7 & 1.2 & 1.4 & 3.8 & 4.6 & 4.1 & 3.07 \\
\hline Cristaldi & 0.7 & 2.7 & 2.8 & 5.1 & 7.6 & 5.4 & 4.64 \\
\hline
\end{tabular}




\section{CONCLUSION}

In this paper, a new PV performance model is introduced, which consists of simple expressions to calculate the SC, OC and MPP operating points at any irradiance and temperature. The proposed equations have solid theoretical foundation and are in very good agreement with the single-diode model. The input data required are always given in the module datasheet, which renders the model generally applicable to any crystalline PV module.

The novelty of the proposed model lies on a new formula to extract the irradiance and temperature coefficients, which rely only on datasheet information and do not require additional measurements, in contrast to the current state-of-the-art. The validity of the model is verified through both simulations in MATLAB and outdoor measurements, while a detailed comparison to other methods found in the literature is included. The conclusion is that the proposed model is the best option for an accurate overall performance estimation of a PV system.

\section{APPENDIX}

\section{A. Multiplication Property of the Lambert W Function}

The definition of the Lambert $W$ function $W\{x\}$ is given by (35) [38], [39], while calculation formulas for positive real values may be found in [27] and [7].

$$
W\{x\} e^{W\{x\}}=x \Leftrightarrow W\{x\}=\ln x-\ln W\{x\}
$$

The Lambert $W$ and the logarithmic functions are of similar nature. Yet, the former has not an explicit multiplication property like the logarithmic product rule, i.e. $W\{x y\}$ cannot be expressed as an explicit function of only $W\{x\}$ and $W\{y\}$, where $x$ and $y$ are independent variables. In particular, if the rightmost version of (35) is applied separately for $W\{x y\}, W\{x\}$ and $W\{y\}$, the resulting equations can be combined into:

$$
W\{x y\}=W\{x\}+W\{y\}+\ln [W\{x\} W\{y\} / W\{x y\}]
$$

In (36), the term $W\{x y\}$ appears at both sides of the equation, so it is not a multiplication property in the conventional sense. It is worth noting, however, that if the last logarithmic term is neglected, then (36) essentially becomes the well-known logarithmic product rule. An equivalent formulation of (36) is given below, derived by substituting (35) for $W\{y\}$ :

$$
W\{x y\}=W\{x\}+\ln y+\ln [W\{x\} / W\{x y\}]
$$

\section{B. Translation Equation of the auxiliary term $w=W\left\{e^{1 / \delta+1}\right\}$}

If (23) and (18) are substituted into the right-hand side of (5) , the following expression is derived after some manipulation:

$$
w=W\{\underbrace{e^{V_{o c} / a+1}}_{x y}\}=W\{\underbrace{e^{1 / \delta_{0}+1}}_{x} \cdot \underbrace{e^{\left(\beta_{\text {Voc }}-1 / T_{0}\right) / \delta_{0} \cdot \Delta T / \lambda T+\ln G}}_{y}\}
$$

In (38), the argument of the Lambert $W$ function has been factorized, permitting application of (37):

$$
w=\underbrace{W\left\{e^{1 / \delta_{0}+1}\right\}}_{w_{0}}+\frac{\beta_{V o c}-1 / T_{0}}{\delta_{0}} \frac{\Delta T}{\lambda T}+\ln G+\ln \underbrace{\left[\frac{W\left\{e^{1 / \delta_{0}+1}\right\}}{W\left\{e^{V_{o c} / a+1}\right\}}\right]}_{L}
$$

The first term in (39) corresponds to $w_{0}$ according to (10), while the last logarithmic term $L$ takes small values and may be simplified as follows. The arguments of the Lambert $W$ function in the nominator, $x$, and denominator, $x y$, take very large values

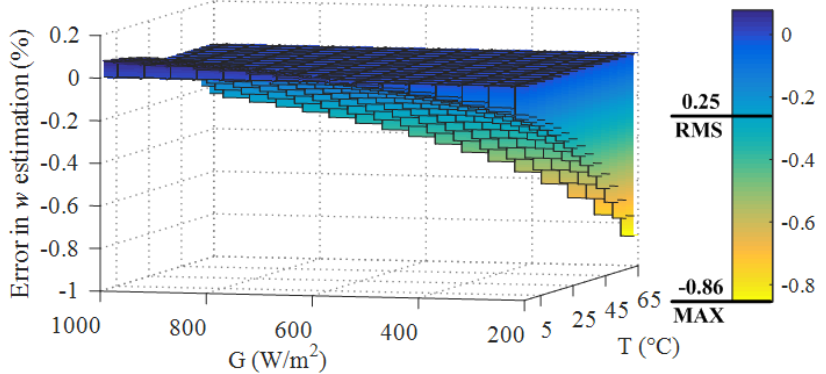

Fig. 9. Error distribution in estimating the auxiliary term $w$ through (21) over the entire range of irradiance and temperature for an indicative PV module considered in Section IV.

due to their large exponents; in this range, the Lambert $W$ function exhibits logarithmic-like behavior. Furthermore, the exponents $1 / \delta_{0}+1$ and $V_{o c} a+1$ are in the same order of magnitude, since the former term is the nominal value of the latter. As a result, the ratio of Lambert $W$ terms in $L$ varies in a very limited range $([0.8,1.5]$ for all scenarios of Section IV), permitting approximation by a ratio of logarithms:

$$
L=\ln \left[\frac{\ln \left(e^{1 / \delta_{0}+1}\right)}{\ln \left(e^{V_{o c} / a+1}\right)}\right]=\ln \left[\frac{1 / \delta_{0}+1}{1 / \delta_{0}+1+\left(\beta_{V o c}-1 / T_{0}\right) / \delta_{0} \cdot \Delta T / \lambda T+\ln G}\right]
$$

This equation may be further simplified considering the first term of the natural logarithm series $\ln (x)=(x-1) / x$ [40], which is sufficiently accurate for arguments close to 1 :

$$
L=\frac{-1}{1 / \delta_{0}+1}\left[\frac{\beta_{V o c}-1 / T_{0}}{\delta_{0}} \frac{\Delta T}{\lambda T}+\ln G\right]
$$

Finally, substituting (41) into (39) for $L$ yields the translation equation of $w$ given by (21). The estimation accuracy of this equation is illustrated in Fig. 9 for one of the study-case PV modules of Section IV. Clearly, the simplifications and assumptions considered in this section hold true and (21) can be reliably used as the translation equation of the term $w$.

\section{Simplification of (32) to (33) for $a_{I m p}$}

The full equation for the temperature coefficient of the MPP current $a_{I m p}$ is given by (32). Yet, the size and complexity of this expression dictates severe simplification in order to acquire a useful form. A first observation is that the temperature effect on $I_{m p}$ is almost the same for the ideal and complete PV models (see Fig. 2), since $R_{s}$ and $R_{s h}$ are not affected by the temperature. Therefore, $a_{I m p}$ could be calculated for the ideal PV model: (7) becomes $I_{m p}=I_{s c}(1-1 / w)$ and if substituted to (32) for STC the second term is crossed out, leading to:

$$
a_{\text {Imp }}=\frac{w_{0}}{w_{0}-1}\left[a_{I s c}+\frac{1}{w_{0} \Delta T}-\frac{1+a_{I s c} \Delta T}{w_{0} \Delta T+\frac{1}{1+\delta_{0}}\left(\frac{\beta_{V o c}-1 / T_{0}}{\delta_{0}} \frac{\Delta T}{\lambda T}+\ln G\right) \Delta T}\right]
$$

This equation is further reduced to (33) after some mathematical manipulation, considering that $\delta_{0}\left(1+\delta_{0}\right) w_{0} \approx 1$ for typical values of $\delta_{0}$ and neglecting the small term $\ln (G)$.

\section{REFERENCES}

W. De Soto, S. A. Klein, and W. A. Beckman, "Improvement and validation of a model for photovoltaic array performance," Sol. 
Energy, vol. 80, no. 1, pp. 78-88, Jan. 2006.

[2]

K. Ding, X. Bian, H. Liu, and T. Peng, “A MATLAB-simulink-based PV module model and its application under conditions of nonuniform irradiance," IEEE Trans. Energy Convers., vol. 27, no. 4, pp. 864872, Dec. 2012.

[3] K. Ding, J. Zhang, X. Bian, and J. Xu, "A simplified model for photovoltaic modules based on improved translation equations," Sol. Energy, vol. 101, pp. 40-52, Mar. 2014.

[4] E. Saloux, A. Teyssedou, and M. Sorin, "Explicit model of photovoltaic panels to determine voltages and currents at the maximum power point," Sol. Energy, vol. 85, no. 5, pp. 713-722, May 2011

[5] L. Cristaldi, M. Faifer, M. Rossi, and S. Toscani, "A simplified model of photovoltaic panel," in Proc. Int. Instrum. Meas. Technol. Conf., Graz, Austria, May 2012, pp. 431-436.

[6] L. Cristaldi, M. Faifer, M. Rossi, and S. Toscani, "An improved model-based maximum power point tracker for photovoltaic panels," IEEE Trans. Instrum. Meas., vol. 63, no. 1, pp. 63-71, Jan. 2014.

[7] E. I. Batzelis and S. A. Papathanassiou, "A method for the analytical extraction of the single-diode PV model parameters," IEEE Trans. Sustain. Energy, vol. 7, no. 2, pp. 504-512, Apr. 2016.

[8] G. Petrone, G. Spagnuolo, and M. Vitelli, "Analytical model of mismatched photovoltaic fields by means of Lambert W-function," Sol. Energy Mater. Sol. Cells, vol. 91, no. 18, pp. 1652-1657, Nov. 2007.

[9] M. L. Orozco-Gutierrez, J. M. Ramirez-Scarpetta, G. Spagnuolo, and C. A. Ramos-Paja, "A technique for mismatched PV array simulation," Renew. Energy, vol. 55, pp. 417-427, Jul. 2013.

[10] J. Accarino, G. Petrone, C. A. Ramos-Paja, and G. Spagnuolo, "Symbolic algebra for the calculation of the series and parallel resistances in PV module model," in Proc. 4th Int. Conf. Clean Electr. Power (ICCEP 2013), Alghero, Italy, Jun. 2013, pp. 62-66.

[11] G. L. Araujo and E. Sánchez, "Analytical expressions for the determination of the maximum power point and the fill factor of a solar cell," Sol. Cells, vol. 5, no. 4, pp. 377-386, Apr. 1982.

[12] M. Fuentes, G. Nofuentes, J. Aguilera, D. L. Talavera, and M. Castro, "Application and validation of algebraic methods to predict the behaviour of crystalline silicon PV modules in Mediterranean climates," Sol. Energy, vol. 81, no. 11, pp. 1396-1408, Nov. 2007.

[13] A. J. Anderson, "Photovoltaic translation equations: A new approach," NREL, Jan. 1996.

[14] B. Marion, B. Kroposki, K. Emery, D. Myers, J. del Cueto, and C. Osterwald, "Validation of a photovoltaic module energy ratings procedure at NREL," NREL, Aug. 1999.

[15] B. Marion, "A method for modeling the current-voltage curve of a PV module for outdoor conditions," Prog. Photovoltaics Res. Appl., vol. 10, no. 3, pp. 205-214, May 2002.

[16] B. Marion, S. Rummel, and A. Anderberg, "Current-voltage curve translation by bilinear interpolation," Prog. Photovoltaics Res. Appl., vol. 12, no. 8, pp. 593-607, Dec. 2004.

[17] ASTM E 1036M - 96, "Standard Test Methods for Electrical Performance of Nonconcentrator Terrestrial Photovoltaic Modules and Arrays Using Reference Cells," Jul. 1996.

[18] D. L. King and P. E. Eckert, "Characterizing (rating) the performance of large photovoltaic arrays for all operating conditions," in Proc. 25th IEEE Photovolt. Spec. Conf., May 1996, pp. 1385-1388.

[19] C. M. Whitaker, T. U. Townsend, J. D. Newmiller, D. L. King, W. E. Boyson, J. A. Kratochvil, D. E. Collier, and D. E. Osborn, "Application and validation of a new PV performance characterization method," in Proc. 26th EEE Photovolt. Spec. Conf., Oct. 1997, pp. 1253-1256.

[20] D. L. King, J. A. Kratochvil, and W. E. Boyson, "Temperature coefficients for PV modules and arrays: measurement methods, difficulties, and results," in Proc. 26th IEEE Photovolt. Spec. Conf., Oct. 1997, pp. 1183-1186.

[21] D. L. King, W. E. Boyson, and J. A. Kratochvil, "Photovoltaic array performance model," Sandia National Laboratories, Aug. 2004.

[22] IEC 60891, "Procedures for temperature and irradiance corrections to measurement I-V characteristics," Dec. 2009.

[23] W. Herrmann and W. Wiesner, "Current-voltage translation procedure for PV generators in the German 1,000 roofs-programme," in Proc. EUROSUN, Freiburg, Germany, Sep. 1996.

[24] W. Zhou, H. Yang, and Z. Fang, "A novel model for photovoltaic array performance prediction," Appl. Energy, vol. 84, no. 12, pp. 1187-1198, Dec. 2007.
[25] J. M. da C. Po, "Assessment of the IEC 60891 norm conversion methods under outdoor test conditions," Master thesis, Faculty of Sciences, University of Lisbon, 2011.

[26] E. I. Batzelis, G. E. Kampitsis, S. A. Papathanassiou, and S. N. Manias, "Direct MPP calculation in terms of the single-diode PV model parameters," IEEE Trans. Energy Convers., vol. 30, no. 1, pp. 226-236, Mar. 2015.

[27] E. I. Batzelis, I. A. Routsolias, and S. A. Papathanassiou, "An explicit PV string model based on the Lambert W function and simplified MPP expressions for operation under partial shading," IEEE Trans. Sustain. Energy, vol. 5, no. 1, pp. 301-312, Jan. 2014.

[28] G. Farivar, B. Asaei, and S. Mehrnami, "An analytical solution for tracking photovoltaic module MPP," IEEE J. Photovoltaics, vol. 3, no. 3, pp. 1053-1061, Jul. 2013.

[29] A. Kuperman, "Comments on 'An analytical solution for tracking photovoltaic module MPP, ” IEEE J. Photovoltaics, vol. 4, no. 2, pp. 734-735, Mar. 2014.

[30] E. I. Batzelis, G. E. Kampitsis, and S. A. Papathanassiou, "Power reserves control for PV systems with real-time MPP estimation via curve fitting," IEEE Trans. Sustain. Energy, no. accepted for publication.

[31] E. Batzelis, G. E. Kampitsis, and S. A. Papathanassiou, "A MPPT algorithm for partial shading conditions employing curve fitting," in Proc. 32th Eur. Photovolt. Sol. Energy Conf. Exhib. (EU PVSEC 2016), Munich, Germany, Jun. 2016.

[32] R. Khezzar, M. Zereg, and A. Khezzar, "Modeling improvement of the four parameter model for photovoltaic modules," Sol. Energy, vol. 110, pp. 452-462, Dec. 2014.

[33] J. Bai, S. Liu, Y. Hao, Z. Zhang, M. Jiang, and Y. Zhang, "Development of a new compound method to extract the five parameters of PV modules," Energy Convers. Manag., vol. 79, pp. 294-303, Mar. 2014.

[34] G. N. Psarros, E. I. Batzelis, and S. A. Papathanassiou, "Partial shading analysis of multistring PV arrays and derivation of simplified MPP expressions," IEEE Trans. Sustain. Energy, vol. 6, no. 2, pp. 499-508, Apr. 2015.

[35] J. D. Bastidas, E. Franco, G. Petrone, C. A. Ramos-Paja, and G. Spagnuolo, "A model of photovoltaic fields in mismatching conditions featuring an improved calculation speed," Electr. Power Syst. Res., vol. 96, pp. 81-90, Mar. 2013.

[36] H. Tian, F. Mancilla-David, K. Ellis, E. Muljadi, and P. Jenkins, "A cell-to-module-to-array detailed model for photovoltaic panels," Sol. Energy, vol. 86, no. 9, pp. 2695-2706, Sep. 2012.

[37] "Surface fitting." [Online]. Available: https://uk.mathworks.com/help/curvefit/surface-fitting.html.

[38] "Lambert W function." [Online]. Available: https://en.wikipedia.org/wiki/Lambert_W_function.

[39] R. M. Corless, G. H. Gonnet, D. E. G. Hare, D. J. Jeffrey, and D. E. Knuth, "On the Lambert W function," Adv. Comput. Math., vol. 5 , no. 4, pp. 329-359, 1996.

[40] "Natural logarithm." [Online]. Available: https://en.wikipedia.org/wiki/Natural_logarithm.

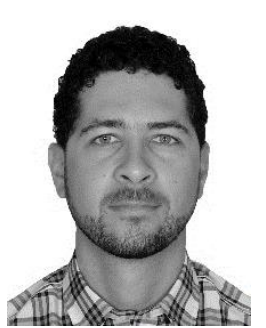

Dr. Efstratios I. Batzelis (S'14-M'17) received the Diploma in electronic \& computer engineering from Technical University of Crete (TUC) in 2009, the M.Sc. degree on energy production \& management from National Technical University of Athens (NTUA) in 2012, and the Ph.D. degree in electrical engineering from the same university in 2016 . He is currently a Research Associate (Postdoctoral Researcher) in Imperial College London.

His current research interests include renewable energy technologies and distributed generation, especially photovoltaics, inverter and power system control. This work was carried out during his $\mathrm{PhD}$ studies at NTUA. 